\title{
Cosmological Implications of Old Galaxies at High Redshifts
}

\author{
J. A. S. Lima*and J. S. Alcaniz ${ }^{\dagger}$ \\ Departamento de Física, Universidade Federal do Rio Grande do Norte, \\ C.P. 1641, 59072-970, Natal, RN, Brazil
}

Received on 21 April, 2001

\begin{abstract}
Old high- $z$ galaxies are important tools for understanding the structure formation problem and may become the key to determine the ultimate fate of the Universe. In this paper, the inferred ages of the three oldest galaxies at high redshifts reported in the literature are used to constrain the first epoch of galaxy formation and to reanalyse the high-z time scale crisis. The lower limits on the formation redshift $z_{f}$ depends on the quantity of cold dark matter in the Universe. In particular, if $\Omega_{m} \geq 0.37$ these galaxies are not formed in FRW cosmologies with no dark energy. This result is in line with the Supernovae type Ia measurements which suggest that the bulk of energy in the Universe is repulsive and appears like an unknown form of dark energy component. In a complementar analysis, unlike recent claims favoring the end of the age problem, it is shown that the Einstein-de Sitter model is excluded at high-z by $3 \sigma$.
\end{abstract}

\section{Introduction}

The existence of old high-redshift galaxies (OHRGs) provides one of the best methods for constraining the age of the Universe [1,2], and, in a similar vein, may be an important key for determining the first epoch of galaxy formation. Recently, different groups [3-7] announced the discoveries of three extremely red radio galaxies at $z=1.175$ (3C 65), $z=1.55$ (53W091) and $z=1.43$ (53W069) with a minimal stellar age of 4.0 Gyr, 3.0 Gyr and 4.0 Gyr, respectively. These discoveries accentuated even further the already classical "age crisis" and gave rise to a new variant of this problem, which could be named the high- $z$ time scale crisis: the underestimated ages of these galaxies contradict the predictions of the standard Einstein-de Sitter model for values of $h>0.45$ [1]. For comparison, if one considers $h=H_{o} / 100 \mathrm{Kms}^{-1} \mathrm{Mpc}^{-1}=0.6$, the age of the Universe predicted by the Einstein-de Sitter CDM model at redshifts $z=1.175$ and $z=1.55$ is $t_{z}=3.35 \mathrm{Gyr}$ and $t_{z}=2.5 \mathrm{Gyr}$, respectively. Such discrepancies become even larger if one takes into account the radio galaxy at $z=1.43$ with a minimal stellar age of 4.0 Gyr. In this case, the predicted age is $t_{z}=2.85 \mathrm{Gyr}$, which yields an age difference greather than $1 \mathrm{Gyr}$. It is still worth noticing that such estimates do not include an incubation time of at least $0.5-1 \mathrm{Gyr}$, which would be more in accordance to the chemo-dynamical evolution

\footnotetext{
*Electronic address: limajas@dfte.ufrn.br

$\dagger$ Electronic address: alcaniz@dfte.ufrn.br
}

models for such objects[8]. As it appears, the problem raised by the ages of these galaxies to the standard CDM model is a strong indication that OHRGs may also constrain appreciably the formation redshift $\left(z_{f}\right)$, i.e., the epoch where the first structures were actually formed. In brief, this is the aim of this paper. In what follows, robust lower limits on $z_{f}$ are derived for open cold dark matter (OCDM) and flat $\Lambda$ CDM models from the estimated ages of the quoted old high- $z$ galaxies. For completeness, we also present a new quantitative approach for analysing the high- $z$ time scale crisis.

Many attempts for determining the first epoch of galaxy formation have been done in the past, and the overall conclusion is that the major period of galaxy formation lies in the redshift interval $1 \leq z \leq 3$, that is, at relatively low redshifts $[9,10]$. Such results are consistent with the simplest cold dark matter (CDM) scenario for structure formation, although its modified version $(\Lambda \mathrm{CDM})$ gave a more natural explanation for the excess of power observed in the galaxy distribution $[11,12]$. In spite of that, recent findings of galaxy candidates at $z>4.0[13-15]$ suggest that our Universe contains more collapsed objects than believed some few years ago, and all this activity at high redshifts cannot be easily accommodated in the CDM scenarios [16]. Since such studies do not exclude the possibility that 
rare events of galaxy formation took place at higher redshifts, the very beginning of the structure formation process remains as one of the most challenging problems in modern cosmology.

Let us first recall that for the class of passively evolving elliptic radio galaxies, almost all amount of gas is believed to be processed into stars in a single episode of star formation, in such a way that the assumption of an instantaneous burst is considered a good approximation for modeling their evolution. For lookback time calculations, an instantaneous burst of star formation means that the age of these OHRGs can be expressed as being almost exactly the time taken by the Universe to evolve from $z_{f}$ to the observed redshift $z_{o b s}$. In the framework of Friedmann-Robertson-Walker (FRW) models with cosmological constant such condition can be translated as

$$
\begin{gathered}
t\left(z_{o b s}\right)-t\left(z_{f}\right)=H_{o}^{-1} \int_{\left(1+z_{f}\right)^{-1}}^{\left(1+z_{o b s}\right)^{-1}} \\
\frac{d x}{\sqrt{1-\Omega_{m}+\Omega_{m} x^{-1}+\Omega_{\Lambda}\left(x^{2}-1\right)}} \geq t_{g},
\end{gathered}
$$

where $\Omega_{m}$ and $\Omega_{\Lambda}$ stand for the present-day matter and vacuum density parameters, respectively. The inequality signal on the r.h.s. of the above expression comes from the fact that the Universe is older than or at least has the same age of any observed structure. Since this natural argument also holds for any time interval, a finite value for the redshift $z_{f}$ provides the lower bound for the galaxy formation allowed by the aged object located at $z_{\text {obs }}$. Models for which $z_{f} \rightarrow \infty$ are clearly incompatible with the existence of the specific galaxy, being ruled out in a natural way.

Before discussing the resulting diagrams, an important point of principle should be stressed. To assure the robustness of the limits on $z_{f}$, we addopt (1) the minimal value for the Hubble parameter, and (2) the underestimated age for all OHRGs. Both conditions are almost self-explanatory. First, as we know, the smaller the value of $H_{o}$, the larger the age predicted by the model and, second, objects with smaller ages are theoretically more easily accommodated, thereby guaranteeing that the models are always favored in the present estimates. For the Hubble parameter we consider the value obtained by the HST Key project which is in agreement with other independent estimates [17], i.e., the round number value $H_{o}=60 \mathrm{~km} / \mathrm{sec} / \mathrm{Mpc}[18,19]$. Indeed, we are being rather conservative since this lower limit was recently updated to nearly $10 \%$ of accuracy ( $h=0.70 \pm 0.07,1 \sigma$ ) by Friedman [19], and the data from SNe also point consistently to $h>0.6$ or even higher [20, 21].

In Fig. 1a we show the $\Omega_{m}-z_{f}$ plane allowed by the existence of these OHRG's for OCDM models. The shadowed horizontal region corresponds to the observed range $\Omega_{\mathrm{m}}=0.2-0.4$ [22], which is used to fix the lower limits on $z_{f}$. As should be physically expected, if the matter contribution increases, a larger value of $z_{f}$ is required in order to account for the existence of these OHRGs within these models. Conversely, for each object, the absolute minimal value of $z_{f}$ is obtained for an empty universe $\left(\Omega_{\mathrm{m}} \rightarrow 0\right)$. In the observed range of $\Omega_{m}$ the allowed values for the formation redshift are unexpectedly high. For example, by considering $\Omega_{\mathrm{m}}=0.3$, as indicated from dynamic estimates on scales up to about $2 h^{-1} \mathrm{Mpc}$ [23], the ages of 3C 65, 53W091 and 53W069 provide, respectively, $z_{f} \geq 6.3, z_{f} \geq 10.5$ and $z_{f} \geq 18$. Such values suggest that these galaxies were formed about 12.5 Gyr ago, or by considering the most recent lower limits for the age of the Universe [24], that such objects were formed when the Universe was $\sim 1.0$ Gyr old. However, since almost all the age of the Universe is at low redshifts $(z=0-2)$, these galaxies may have been formed nearly at the same epoch, regardless of their constraints on the redshift space.
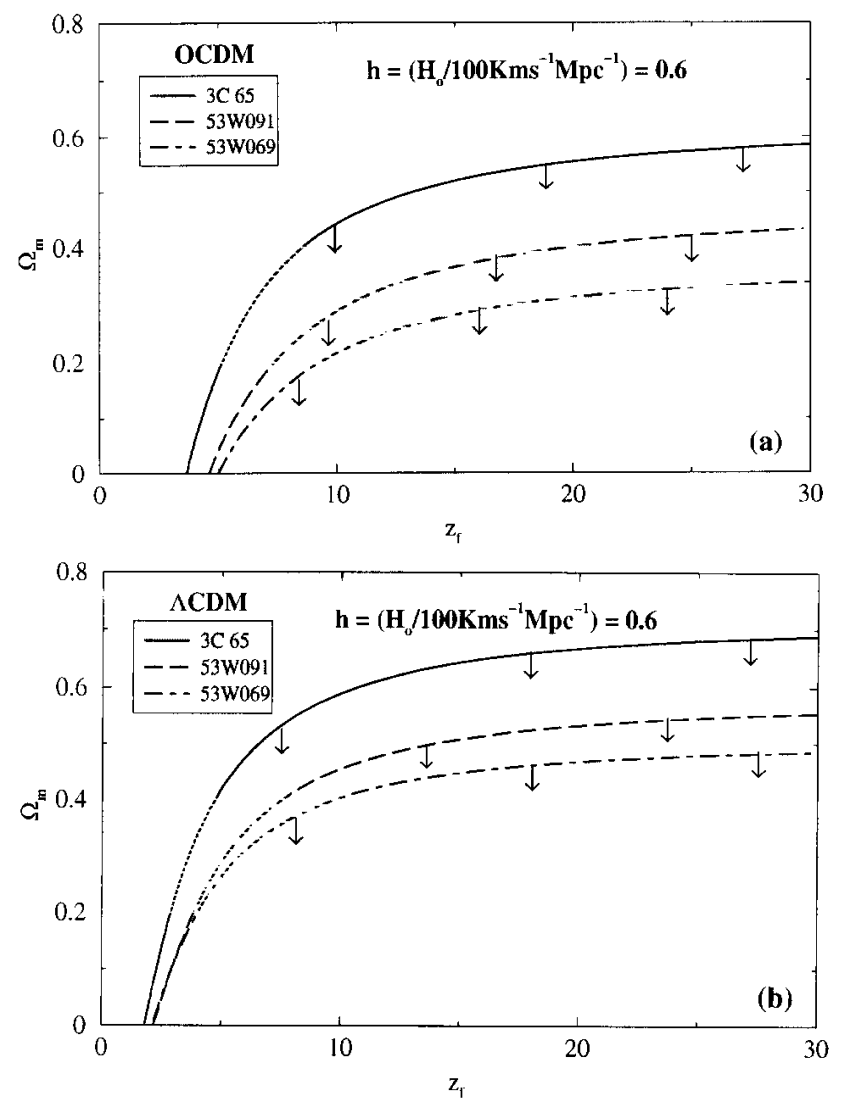

Figure 1. The $\Omega_{m}-z_{f}$ plane allowed by the existence of OHRG's in the framework of OCDM (Panel a) and $\Lambda$ CDM (Panel b). The shadowed horizontal region corresponds to the observed range of $\Omega_{\mathrm{m}}$. The arrows delimit the available parameter space. The curves are also defined by the underestimated values of $t_{g}$ and the indicated lower limit of $H_{o}$. For a given value of $\Omega_{m}$, we see that the most restrictive limit is provided by the radio galaxy 53W069.

Fig. 1b shows similar plots for flat $\Lambda$ CDM models. In this case, the effect of the equation of state associated to the "vacuum medium" $\left(p_{v}=-\rho_{v}\right)$ is to 

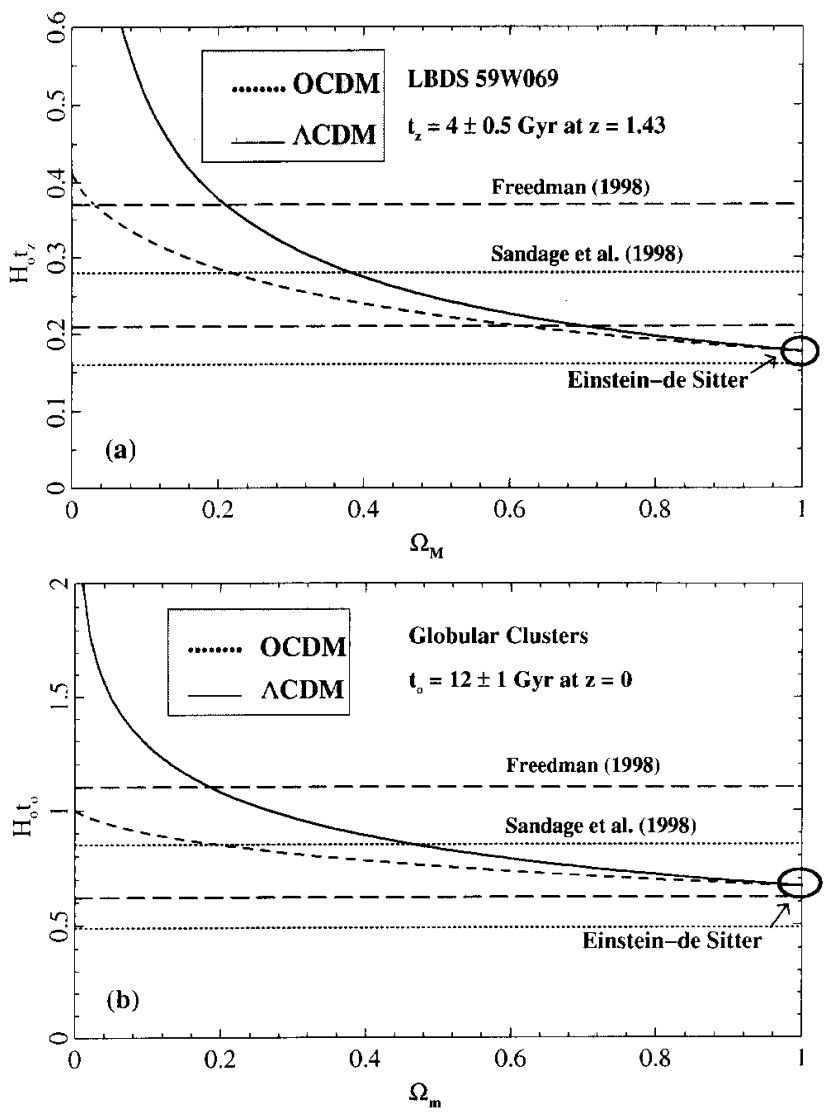

Figure 2. $H_{o} t_{z}$ as a function of $\Omega_{\mathrm{m}}$. Dotted lines indicate the $\pm 2 \sigma$ limits of the age-parameter for $h=0.55 \pm 0.05$ [27] whereas dashed lines indicate the $\pm 2 \sigma$ limits for $h=$ $0.7 \pm 0.1$, the value obtained by the HST Key Project [18] and by other independent estimates $[17,20]$. Note that for $h=0.7 \pm 0.1$ the Einstein-de Sitter case (indicated in the panels) is off by $3 \sigma$ for the analysis of OHRGs. For sake of completeness, Panel b shows similar analysis for globular clusters - see also [19].

accelerate the cosmic expansion. In particular, this means that the lookback time between $z_{o b s}$ and $z_{f}$ is larger than in the standard scenario and, therefore, the galaxy formation process may start relatively late in comparison to the corresponding OCDM model. For example, if the density parameter is around the central value, $\Omega_{\mathrm{m}}=0.3$, the ages of $3 \mathrm{C} 65,53 \mathrm{~W} 091$ and 53W069 restrict the formation redshift by $z_{f} \geq 3.6$, $z_{f} \geq 5.2$ and $z_{f} \geq 5.8$, respectively. The value of $z_{f}$ is also proportional to the quantity of dark matter. As one may check, in the limiting case of a universe dominated only by dark energy, the lower limit is $z_{f} \geq 2$. It is worth noticing that for $\Omega_{\mathrm{m}} \geq 0.37$, the lower limit inferred from the age of 59W069 is $z_{f} \rightarrow \infty$. This result is consistent with recent studies based on the age-redshift relation[2, 25], and means that the standard cosmological model with $\Omega_{\mathrm{m}} \geq 0.37$ and $h \geq 0.6$ is (beyond doubt) incompatible with the existence of this galaxy. We also stress that the present constraints on the formation redshift are indeed rather conservative since the lower limit on $H_{o}$ has been considered in all
Limits to $z_{f}$ : OCDM and $\Lambda$ CDM models

\begin{tabular}{clll}
\hline Galaxy & $\Omega_{\mathrm{m}}$ & $\frac{\text { OCDM }}{z_{f}}$ & $\frac{\Lambda \text { CDM }}{z_{f}}$ \\
\hline 3C 65:......... & 0.2 & $\geq 5.0$ & $\geq 3.0$ \\
& 0.3 & $\geq 6.3$ & $\geq 3.6$ \\
& 0.4 & $\geq 8.6$ & $\geq 4.8$ \\
53 W091:....... & 0.2 & $\geq 7.4$ & $\geq 3.8$ \\
& 0.3 & $\geq 10.5$ & $\geq 5.2$ \\
& 0.4 & $\geq 20.7$ & $\geq 7.4$ \\
53 W069:....... & 0.2 & $\geq 9.3$ & $\geq 3.9$ \\
& 0.3 & $\geq 18.0$ & $\geq 5.8$ \\
& 0.37 & $\rightarrow \infty$ & $\geq 10$ \\
\hline
\end{tabular}

the estimates. Finally, we stress that these lower limits on $z_{f}$ provide a new theoretical evidence that galaxies are not uncommum objects at very large redshifts, say, at $z>5$, and also reinforce the interest on the observational search for galaxies and other collapsed objects within the redshift interval $5 \leq z \leq 10$ (For a discussion about the influence of an arbitrary equation of state $p_{x}=\omega \rho_{x}(\omega \geq-1)$ on the redshift formation, see [26]).

Another important implication of age dating of high- $z$ objects is on the expanding age of the Universe. As before, a crucial point is concerned to the measurements the Hubble parameter. Recently, Sandage et al. [27] argued for the end of the age crisis by concluding that there is no time scale crisis in cosmology if $H_{o}=55 \pm 5 \mathrm{kms}^{-1} \mathrm{Mpc}^{-1}$ as inferred by the Hubble diagram of 52 fiducial SNe Ia. For $h=0.55 \pm 0.05$ and by taking $t_{o}=12 \pm 1(1 \sigma)$ as the median value for the estimated ages of globular clusters [19], we find $H_{o} t_{o}=0.67 \pm 0.09$ which is clearly compatible with the prediction of the Einstein-de Sitter model $\left(t_{o}=\frac{2}{3} H_{o}^{-1}\right)$. Moreover, even by adding $1 \mathrm{Gyr}$ as incubation time [28] we find $H_{o} t_{o}=0.73 \pm 0.1$ which is also compatible with the standard flat CDM model. However, what can be said about a similar analysis at high redshift?

In Fig. 2a we assume 0.5 Gyr as incubation time for the LBDS 53W069 and show the dimensionless product $H_{o} t_{z}$ as a function of $\Omega_{\mathrm{m}}$. Two different cases are illustrated: the standard CDM and the flat $\Lambda$ CDM models. Dotted lines indicate the $\pm 2 \sigma$ limits of the age-parameter by considering $h=0.55 \pm 0.05$ whereas dashed lines indicate the $\pm 2 \sigma$ limits for $h=0.7 \pm 0.1$, the value obtained by the $H S T$ Key Project [18] and by other independent estimates [17, 20]. At this redshift $(z=1.43)$, the prediction of the flat matter-dominated model is $H_{o} t_{z} \leq 0.17$ whereas for $h=0.55 \pm 0.05$ this galaxy yields $H_{o} t_{z}=0.22 \pm 0.03$, which rules out the Einstein-de Sitter case by $\sim 2$ standard deviations. In the case of the LBDS 53W091 (at $z=1.55$ ), the prediction of the Einstein-de Sitter model is $H_{o} t_{z} \leq 0.16$, and similar analysis provides $H_{o} t_{z}=0.20 \pm 0.03$. As expected, such discrepancies become even larger if one 
considers $h=0.7 \pm 0.1$. In this case, we find for the LBDS 53W091 and LBDS 53W069 $H_{o} t_{z}=0.29 \pm 0.04$ and $H_{o} t_{z}=0.25 \pm 0.05$, which excludes the Einstein-de Sitter model by $2 \sigma$ and $3 \sigma$, respectively. Therefore, if the estimated age of these objects are correct, we may conclude, at light of the above analysis that there is a high- $z$ time scale crisis in the standard cosmology, even by considering low values for the Hubble parameter. Naturally, such a result is in agreement with the latest measurements from Supernovae type Ia which point to the existence of a repulsive energy component dominating the bulk of matter-energy in the Universe, thereby implying that the Universe is necessarely older than the age predicted by the Einstein-de Sitter model.

\section{Acknowledgements}

This research was partially supported by the Conselho Nacional de Desenvolvimento Científico e Tecnológico (CNPq), Pronex/FINEP. (no. 41.96.0908.00) and FAPESP (no. 00/06695-0)

\section{References}

[1] L. M. Krauss, Astrophys. J. 480, 466 (1997)

[2] J. S. Alcaniz and J. A. S. Lima, Astrophys. J. 521, L87 (1999)

[3] S. J. Lilly \& M. S. Longair, Mon. Not. R. Astr. Soc. 211, 833 (1984)

[4] A. Stockton, M. Kellogg and S. E. Ridgway, Astrophys. J. 443. L69 (1995)

[5] J. S. Dunlop et al., Nature, 381, 581 (1996)

[6] H. Spinrard, A. Dey, D. Stern, J. S. Dunlop, J. A. Peacock, R. Jimenez, and R. Windhorst, Astrophys. J. 484, 581 (1997)

[7] J. S. Dunlop, in The Most Distant Radio Galaxies, ed. H. J. A. Rottgering, P. Best, \& M. D. Lehnert, Dordrecht: Kluwer, 71 (1999)

[8] R. Jimenez, A. C. S. Friaça, J. S. Dunlop, R. J. Terlevich, J. A. Peacock, L. A. Nolan, Mon. Not. R. Astr. Soc. 305, L16 (1999)
[9] L. L. Cowie, E. M. Hu \& A. Songaila, Nature 377, 603 (1995)

[10] S. E. Zepf, Nature 390, 377 (1997)

[11] S. J. Maddox, G. Efstathiou, W. J. Sutherland \& J. Loveday, Mon. Not. R. Astr. Soc., 242, 43P (1990)

[12] Efsthatiou G., Sutherland W. \& Maddox, S. J., Nature 348, 705 (1990)

[13] E. M. Hu \& R. G. McMahon, Nature 382, 231 (1996)

[14] A. Dey, H. Spinrad, D. Stern, J. R. Graham \& F. H. Chaffee, Astrophys. J 498, L93 (1998)

[15] H.-W. Chen, K. M. Lanzetta \& S. A. S. Pascarelle, Nature, 398, 586 (1999)

[16] A. Kashlinsky \& R. Jimenez, Astrophys. J. 474, L81 (1997)

[17] R. Giovanelli et al., Astrophys. J. 477, L1 (1997)

[18] W. L. Freedman, in Proceedings of the 18th Texas Symposium on Relativistic Astrophysics, edited by A. Olinto, J. Frieman, and D. Schramm, World Scientific, (1998)

[19] W. L. Freedman, Phys. Rept. 333, 13 (2000)

[20] S. Perlmutter, G. Aldering, G. Goldhaber, et al., Astrophys. J. 517, 565

[21] A. G. Riess et al., Astron. J 116, 1009 (1998)

[22] A. Dekel, D. Burstein and S. White, In Critical Dialogues in Cosmology, edited by N. Turok World Scientific, Singapore (1997)

[23] R. G. Calberg et al., Astrophys. J. 462, 32 (1996)

[24] E. Carretta et al., Astrophys. J. 533, 215 (2000); L. M. Krauss, Phys. Rep., 333, 33 (2000)

[25] J. A. S. Lima \& J. S. Alcaniz, Mon. Not. R. Astr. Soc. 317, 893 (2000)

[26] J. S. Alcaniz \& J. A. S. Lima, Astrophys. J. 550, L133

[27] A. Sandage, G. A. Tammann and A. Saha, Phys. Rept. 307, 1 (1998)

[28] J. P. E. Peebles, Publ. Astron. Soc. Pac. 111, 274 (1999) 